\title{
Towards a Process Model of Temporal Generalization
}

\author{
Florian Klapproth
}

\section{1 \\ Introduction}

There are just a few studies concerned with response time in stimulus generalization. For example, Pierrel and Murray (1963) showed that when people compared a given standard with stimuli differing from the standard only in weight magnitude, the decision time increased the smaller the difference was between the standard and the comparison stimulus. Desiderato (1964) presented participants with stimuli repeatedly after a 12-s interstimulus interval in the training phase. The participants were required to release a button as soon as they experienced the stimuli. In the subsequent testing phase, the same stimuli were presented after intervals that were either shorter than, longer than, or the same as the initial 12-s interstimulus interval. The time it took to release the finger from the button increased as a function of the difference between the standard and the comparison intervals. The task that was applied by Desiderato is commonly called a temporal-generalization task.

In temporal generalization, the participants are usually presented with a standard duration after which a series of stimuli of different durations follows, and the participant's task is to judge whether each of the successive durations matches the standard duration or not, by making a same or different response. A typical result is that on most of the trials when it is presented the standard duration is reliably identified (that is, a same response is given). On some trials, however, people will make a same judgment even though a non-standard duration had been presented. Hence, people are likely to generalize a same judgment to stimuli that are shorter or longer than the standard, and the probability of making a same response after the presentation of a non-standard comparison stimulus increases as the difference between the non-standard comparison and the standard itself becomes smaller. When plotting the proportions of same responses against the stimulus durations presented a gradient of generalization will result. The steeper the gradient is, the more accurately the participants have identified the standard amongst the other durations of the series. Usually, the gradient is slightly asymmetrical, with more same 
responses occurring at stimuli longer than the standard (e.g., Wearden, 1992; Wearden, Denovan, Fakhri, \& Haworth, 1997; Weisman et al., 1999).

When comparing the standard duration with a comparison duration in a temporal-generalization task, people are supposed to relate the durations in a way suggested by Wearden (1992). Wearden assumed that people decide for the sameness of both intervals if $a b s(s-t) / t<b$, with $s$ being the standard duration, $t$ being the comparison duration, and $b$ representing a decision threshold. Wearden found that a decision according to that rule fitted empirical data to a high degree of precision, when $s$ and $b$ were variables with random error, but with fixed mean and standard deviation, and $t$ was an error-free variable, changing its value only depending on the comparison duration.

The selection of the parameters used in the Wearden's (1992) model was inspired by the scalar timing theory (Gibbon \& Church, 1984; Gibbon, Church, \& Meck, 1984), which offers an information processing model of the timing processes in humans and animals. The model comprises a clock stage, a memory stage, and a comparison stage. It assumes that when the duration of a stimulus has to be judged, a clock-like mechanism produces pulses, and these pulses are added and temporarily stored in an accumulator. The number of pulses summed in the accumulator represents the duration to be judged. When an interval is regarded as important (like the standard in a temporal generalization task), it should not only be stored temporarily, but for a longer period (at least for the duration of the task at hand). To account for this, the scalar timing theory posits a longer-term (or reference) memory. When making a comparison between the standard and a just-presented duration, the scalar timing theory presumes that a sample of the standard represented in reference memory enters the comparison stage and is related to the current comparison interval. The representation of the standard is assumed to be noisy, so that it may vary from trial to trial due to variations in encoding the standard or as a result of transferring the standard to the comparison stage (Gallistel \& Gibbon, 2000; Gibbon, 1991).

The scalar timing theory specifies the stages that temporal information has to pass through before a decision can be made. However, the model does not describe or predict the time one needs to make a temporal decision. An attempt to characterize the time course of temporal decisions was made by Klapproth and Müller (2008). In their experiments, participants carried out a temporal generalization task, where one group was encouraged to make a temporal judgment as quickly as possible, whereas the other group was not instructed to make a fast judgment. Klapproth and Müller were interested in investigating the relationship between the duration of the comparison stimuli and the timing of the participants' responses, and they expected that when the 
speed of responses is emphasized, it would result in reliable estimates of the time needed to judge the duration of a stimulus.

\section{A Theoretical Model for Response Times in Temporal Generalization}

Klapproth and Müller (2008) suggested a model of the relationship between $a b s(s-t) / t$, the response time $(R T)$, and the stimulus duration $(t)$, which is depicted in Figure 7.1. Response time is defined as the interval between stimulus onset and the participant's response.

The time of the decision, and, hence, the response time $(R T)$ for a correct decision, can be predicted by the relation of the ratio $a b s(s-t) / t$ to the criterion $b$. The ratio $a b s(s-t) / t$ decreases as the duration of the stimulus presentation $(T)$ increases, and reaches a minimum at the instant $T_{\mathcal{S}}$, where $t=s$. A further increase of the stimulus duration $T$ would cause an increase of $a b s(s-t) / t$ which eventually approximates 1 , since the longer $t$ is, the more similar will be the numerator and the denominator of the ratio. As can be seen in Figure 7.1, the function $f(T)=a b s(s-t) / t$ crosses the threshold $b$ twice. The interval between the two points of intersection, $\left(T_{1} ; b\right)$ and $\left(T_{2} ; b\right)$, marks the duration values for which a same response is expected to occur

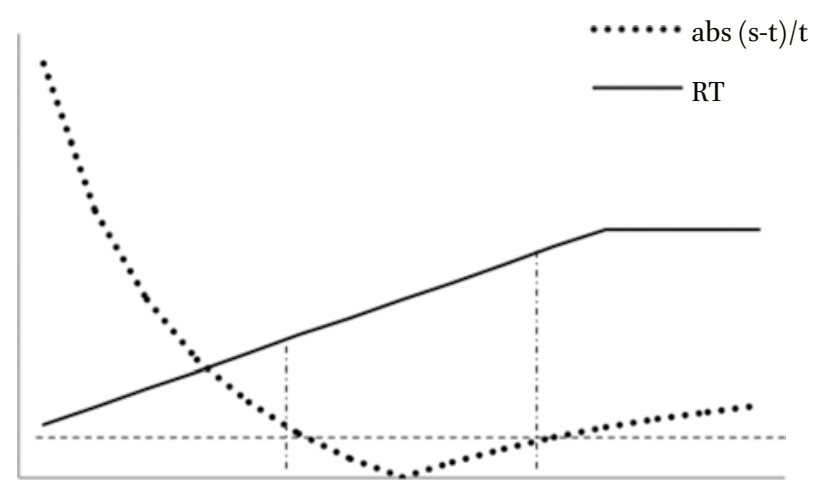

Stimulus duration $(\mathrm{T})$

FIGURE 7.1 The relation between abs $(s-t) / t$, response time $(R T)$, and stimulus duration $(T)$. The dotted curved line represents abs $(s-t) / t$ as a function of $T$, the solid line represents the response times as a function of $T$, and the vertical lines represent the time values $T_{1}$ and $T_{2}$. The horizontal dotted line shows $b$. MODIFIED AFTER KLAPPROTH AND MÜLLER (2008). 
Response times for same responses should increase with increasing stimulus duration, as the participants cannot make a same decision until after the stimulus has ended, because the stimulus might always go on for longer. For different responses, this is true up until the standard duration (plus some additional time) has been arrived at, then response times should flatten out, as the participant may have enough information to make the response before the entire stimulus has ended.

Based on these assumptions, Klapproth and Müller (2008) proposed a temporal decision rule according to which a decision for the sameness of two intervals is expected if $T_{1} \leq T \leq T_{2}$, where $T$ is the duration of the stimulus just presented. A decision for the difference between two intervals is expected if $T<T_{1}$ or $T>T_{2}$. At $T_{1}, t$ is smaller than $s$, so that $t\left(T_{1}\right)=s /(1+b)$, where $t\left(T_{1}\right)$ is the mental representation of $T_{1}$. The reverse relation between $t$ and $s$ can be observed at $T_{2}$, where $t>s$, so that $t\left(T_{2}\right)=s /(1-b)$.

The response times should be linearly related to the stimulus duration for stimulus durations that are shorter than or equal to $T_{2}$, whereas it was presumed that constant response latencies should occur for durations that are longer than $T_{2}$. Similar assumptions have been made, for instance, by Balcı and Simen (2014)

\subsection{Gradients of Temporal Generalization}

In the study conducted by Klapproth and Müller (2008) both proportions of same responses as well as response times were recorded and analyzed. The gradients depicted below (Figure 7.2) reflect the proportions of same responses obtained at different stimulus durations in the first experiment of this study. The standard stimulus duration was $1000 \mathrm{~ms}$. When the participants responded quickly, a leftward shift of the gradient occurred, compared to the gradient obtained from the participants who were not urged to respond quickly.

Klapproth and Müller (2008) presumed that the leftward shift of the gradient was the result of a change of the standard represented in memory due to the truncation of the longest intervals of the series at hand, and referred to the adaptation-level theory (Helson, 1948, 1964; Thomas, 1993) as a potential source of explanation of the gradient's shift. According to this theory, the mean value of all stimulus durations presented within an experiment provides a frame of reference for making a decision about the duration of each comparison stimulus. In temporal-generalization tasks normally used, the mean of all durations equals (or is near to) the standard duration. In the speed conditions of the Klapproth and Müller (2008) study, however, the participants may not have perceived the full duration of the longest comparison stimuli of the series 


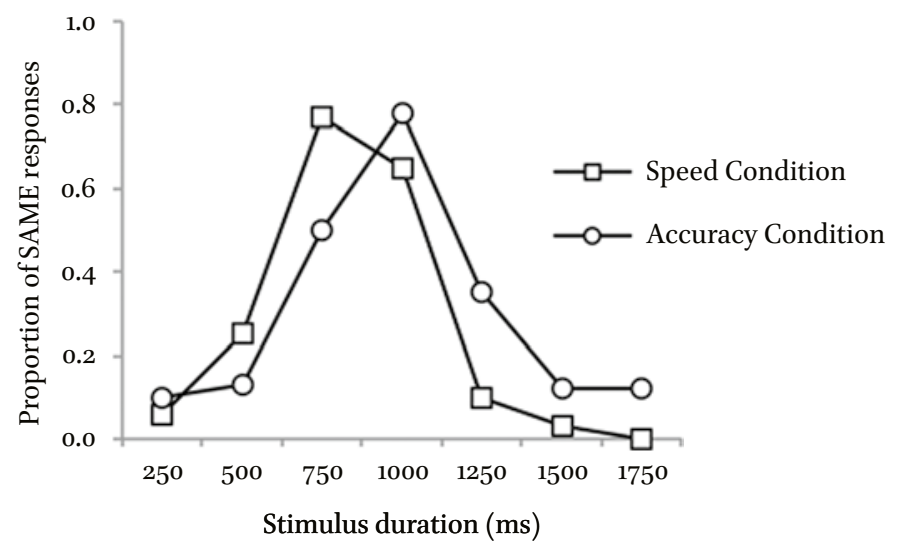

FIGURE 7.2 Temporal generalization gradients obtained from Experiment 1 of the Klapproth and Müller (2008) study. MODIFIED AFTER KLAPPROTH AND MÜLLER (2008).

because they were able to make a response prior to the cessation of these long stimuli. Therefore, the mean of the durations experienced by the participants may have been smaller in the speed conditions than in the control conditions. Klapproth and Müller concluded that the participants in the speed conditions related the comparison durations not to the standard itself but to the mean of all experienced durations, a value which apparently was smaller than the nominal standard duration. Hence, the gradient of the speed conditions had a leftward shift, whereas the gradient of the control conditions was not shifted.

\subsection{Response Times}

The response times were much shorter in the speed groups than in the control groups, and the time taken for same responses increased linearly with stimulus duration, whereas the time taken for different responses grew proportionally with stimulus duration but then remained approximately constant at stimulus durations longer than the standard. Figure $7 \cdot 3$ shows the standardized response times obtained from the Klapproth and Müller (2008) experiments. Standardized response times were response times minus the intercept of the linear regression of response times, divided by the standard duration. In the Klapproth and Müller study, the standard durations used were $75^{\circ}, 1000$, and $125^{\circ}$ ms. The standardization has therefore resulted in response times that were close to the identity line (which is, in Figure $7 \cdot 3$, the dotted diagonal), and response times would have been larger without standardization.

However, some unexpected deviations from the predicted RT model occurred. First, same responses at the longest durations were given faster than 

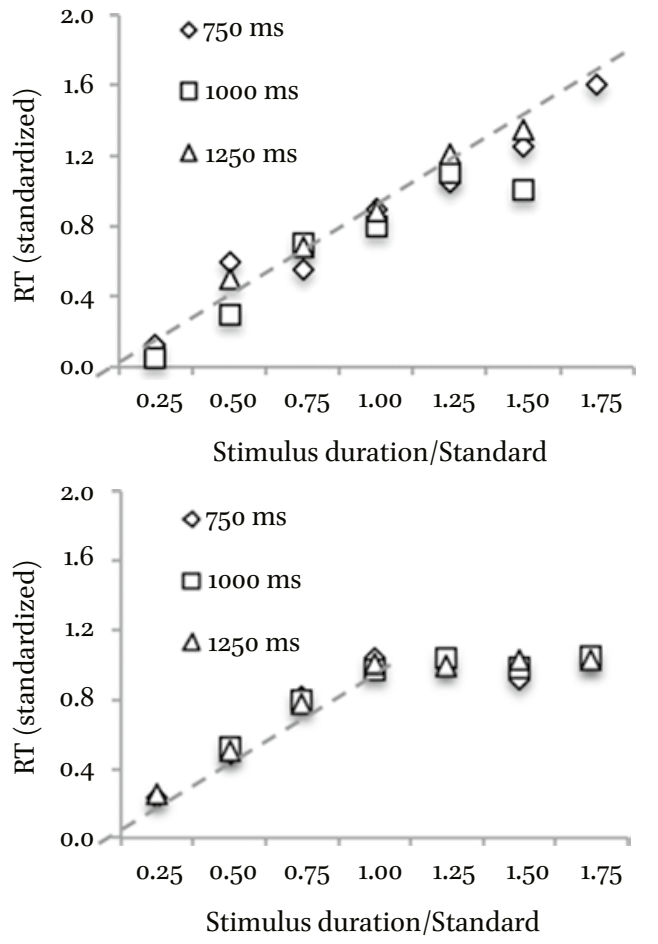

FIGURE 7.3 Standardized response times, plotted against comparison stimulus duration divided by the corresponding standard. Data are shown separately for the conditions with the 750, 1000, and 1250 ms standard. The dotted grey line in the background represents the expected standardized RT. Upper panel: response times for "same" responses; lower panel: response times for "different" responses. MODIFIED AFTER KLAPPROTH AND MÜLLER (2008).

same responses at shorter durations. This might have been done because these responses were merely "fast guesses". Moreover, the number of same responses given at the longest durations was quite small, so that the data points at these durations were rather unreliable (and, therefore, might not reflect a stable trend). Second, different responses were somewhat slower than same responses before the turning point $\left(T_{2}\right)$ occurred. According to the literature pertinent to this phenomenon (see, for example, Farrell, 1985), I will call this result a fast-same effect.

\subsection{The Fast-same Effect}

How could same responses be faster than different responses? This is an "old" question since there are quite a lot of results and models being related to this question, of which some have been proven to be more successful than others 
(Farell, 1985). For example, fast-same responses have been discussed as a matter of encoding facilitation (Nickerson, 1975; Posner, Klein, Summers, \& Buggie, 1973; Posner \& Snyder, 1975). The time taken to process a stimulus might depend not only on the stimulus presented, but also on preceding stimuli. In particular, stimulus repetition might facilitate the encoding of latter occurrences of that stimulus, perhaps by priming neural pathways. Since in temporal generalization (and, in particular, in the experiments we conducted) the target stimulus (i.e., the standard) had been presented more frequently than other stimuli, encoding facilitation might be a cause for the fast-same effect observed. However, in our experiments fast-same responses were given mainly at intervals shorter than the initial standard, and repetition of the standard would not have facilitated the encoding of stimuli shorter than the standard.

According to Krueger's noisy-operator model (Krueger, 1978), comparing a test stimulus with a target might involve counting the mismatches between a number of stimulus attributes. Due to noise in the comparison process, some mismatch counts are indecisive, leading to a rechecking process. The probability of rechecking is supposed to be greater for different responses than for same responses, thus different response times will on average be longer same response times. However, in temporal generalization, only one feature (the duration) is relevant for comparison.

Despite there being some approaches aiming at explaining the fast-same effect, there seems to be no explaining model that especially covers this phenomenon with timing data. My approach to resolve this problem is based on fairly simple assumptions. The first assumption is that response time depends on the subjective probability for an event to occur in choice tasks. The more likely an event is to occur, the more "prepared" should a participant be, the higher should be his or her "readiness" to respond to that event.

Figure 7.4 illustrates the probability model. Suppose there are two alternatives a participant has to select from, for example, the choice between a same response and a different response. In temporal generalization, the initial (subjective) probability for the occurrence of the standard (and, hence, for responding with same) is approximately the same as the probability for a different response, for the frequency of standard and non-standard stimuli presented to the participant is more or less equal. As time goes by, that is, as the stimulus is being presented, the probability for a same response increases whereas it decreases for a different response. The reason is as follows: short durations are no longer subject of choice when the stimulus presentation has passed those durations. Hence, the number of non-standards that still can be chosen reduces as the stimulus presentation prolongs. The probability for a same response grows to its maximum when the presented duration equals the 


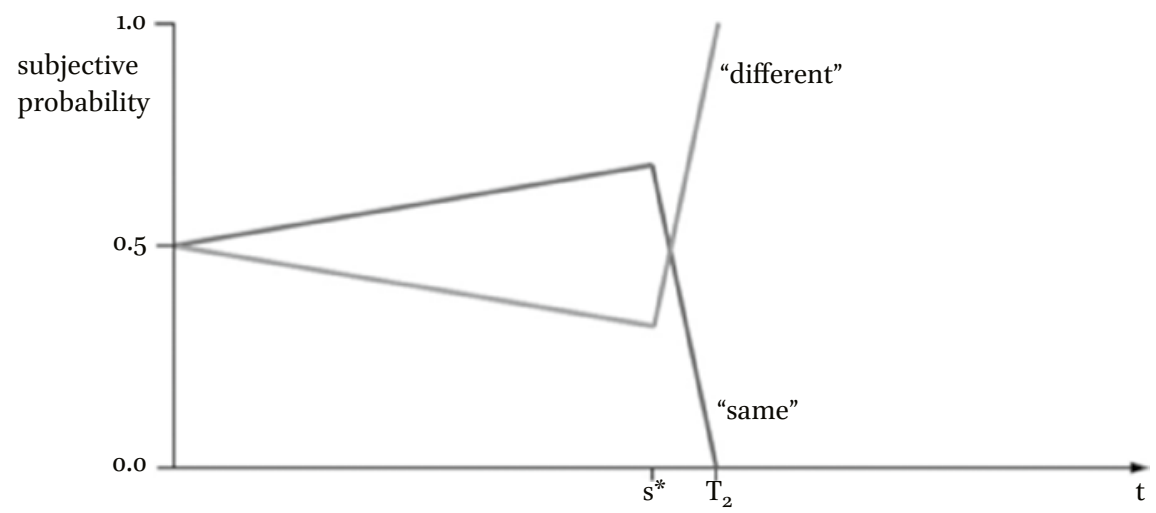

FIGURE 7.4 A model of subjective probability of same or different responses in temporal generalization, dependent on the duration of the comparison interval $t$.

(subjective) standard duration, $t=s^{*}$. After that instant, the probability for a same response decreases rapidly and reaches its minimum $(p=0)$ shortly after passing the threshold $T_{2}$.

With respect to subjective probability, the speed of responding with same should increase the closer the current duration is to $s^{*}$. Accordingly, the speed of responding with different should decrease. As can be inferred from Figure 7.4, same responses ought to be faster than different responses for intervals shorter than the (subjective) standard. Whereas they should be fairly equal at very short durations, our data suggest that even at very short intervals (e.g., $100 \mathrm{~ms}$ ), the same-different difference occurred. How could this finding be explained?

The answer may lie in the "dynamic nature" of $s^{*}$, which leads to my second assumption (see Figure 7.5). The scalar timing theory suggests that same responses are made when $t=s^{*}$. If $s^{*}<s$, same responses will be made at durations shorter than the standard. That is, same responses made at intervals shorter than the standard (even at very short intervals) might be done because in those trials the subjective value of the standard was very small, too. Different responses, however, do not "need" a change of the value of $s^{*}$ to be executed at short intervals. They can be made even if $s^{*}=s$.

According to the probability model, both same responses and different responses can be sped due to the increased "preparedness" of the participant. Consider first the case that a same response is given which should happen when $t=s^{*}$. The line indicating same responses in Figure 7.5 shows that the subjective probability for a same response is higher than that for a different response. Thus, response time will be relatively short. Now imagine that the participant's memory sample of the standard is the same but the current interval is longer than $s^{*}$. As can be seen in Figure 7.5, the subjective probability for 


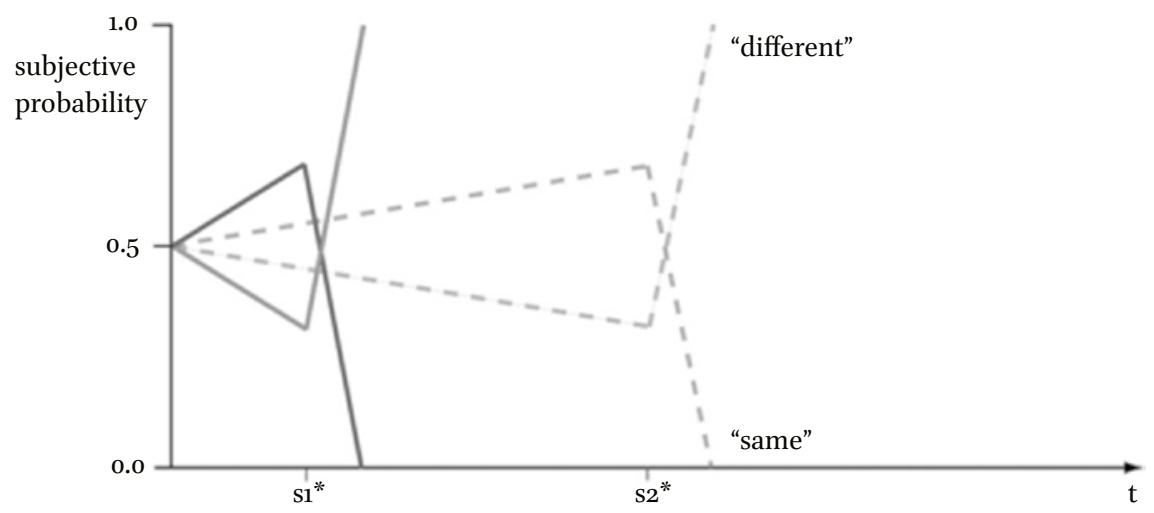

FIGURE 7.5 The development of subjective probability of same or different responses in temporal generalization, dependent on the mental representation of the standard duration $s^{*}$.

a same response will decrease and—accordingly—will increase for a different response. In that case, a different response will occur which is made rapidly because it is considered to be very likely. (This would also be the case when intervals longer than the subjective standard are truncated.) Now suppose a third case in which the current interval is shorter than the subjective standard $s^{*}$. A different response would occur which takes more time to be executed than a same response would do at the same interval, for a same response should be expected to a higher degree than a different response.

This example illustrates that there might be three classes of responses: fast-same responses, fast-different responses, and slow-different responses. Fast-same responses should be made when $t=s^{*}$, fast-different responses should be made when $t>s^{*}$, and slow-different responses should be made when $t<s^{*}$. To yield a fast-same effect, fast-same responses must overweigh fast-different responses (i.e., same responses must on average be faster than different responses given at a certain stimulus duration).

Compared to different responses, same responses were observed to need more time to be given at intervals larger than the standard. This was presumably the case because different responses could be made without completely processing stimulus duration of larger intervals whereas same responses could be made only after the whole stimulus was experienced. Therefore, the fastsame effect was observed only for intervals shorter than the standard.

\section{$2.4 \quad$ Another Experiment}

In an experiment conducted by Klapproth and Wearden (2011), a temporalgeneralization task was used where the standard duration was not at the center of all durations presented, but either the smallest or the largest value of 
the durations. The participants were assigned to four conditions, half of which were speed conditions, the remaining half control conditions. It was expected that only durations longer than the standard were truncated when participants were urged to respond quickly. Therefore, a shift should occur only in the speed condition with long durations and not in the speed condition with short durations. Figure 7.6 shows the gradients obtained from the Klapproth and Wearden experiment (dark-grey: speed conditions; light-grey: accuracy conditions).

As hypothesized, a shift of the gradients towards shorter durations only occurred when the participants were presented with stimulus durations that were either the same as or longer than the standard duration. However, when comparison durations were shorter than or equal to the standard, no shift was observed. Therefore, experiencing stimulus durations that are longer than the standard appears to be necessary to produce the shift in response gradients during temporal generalization under time pressure. Figure 7.7 shows the response times obtained from this experiment.

As Figure 7.7 shows, response times for same responses increased linearly with stimulus duration, with some deviations from linearity occurring at the shortest and longest stimulus duration which might be considered outliers since each of these data points represented only a few responses ( 1 or 3 , respectively). Like with the response times of the Klapproth and Müller (2008) experiment, different responses increased up to a certain point and then maintained their amount. What is striking here is that response times of the "short" condition were apparently longer at the $1000 \mathrm{~ms}$ duration than response times of the "long" condition. This was true for both same responses and different responses. In regard to the same responses, however, the difference in response times

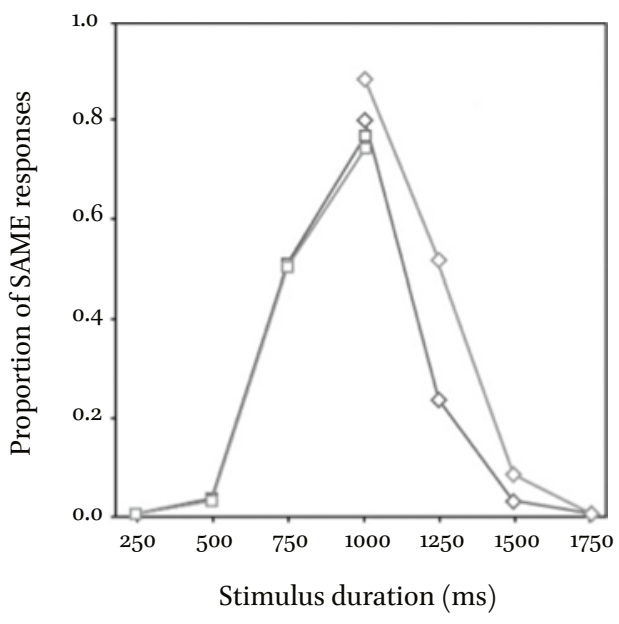

FIGURE 7.6 Temporal-generalization gradients obtained from Experiment 2 of the Klapproth and Wearden (2011) study. MODIFIED AFTER KLAPPROTH AND WEARDEN (2011). 
between both conditions was not statistically significant, $t(28)=1.93, p=.07$, $d=0.81$. Yet, the difference between the "short" and the "long" condition with respect to different responses was much more substantial, $t(26)=3.73, p<.001$, $d=1.41$. The turning point at which response times changed from increasing to keeping their value constant occurred earlier in the "short" condition than in the "long" condition. This might be attributed to a difference in $s^{*}$ between both conditions. Remember that in the "long" condition longer intervals had been experienced than in the "short" condition (although the longest might have been truncated). According to the adaptation-level theory, the reference value for comparisons with current duration values should be larger in the "long" condition than in the "short" condition. Therefore, the instant to make a different response at intervals larger than the subjective standard should be later in the "long" condition than in the "short" condition.

Furthermore, the fast-same effect observed in the Klapproth and Müller (2008) experiment did not occur in this experiment (except for the few data points at the shortest duration). On the contrary, different responses in the "long" condition were on average faster than same responses, whereas in the "short" condition, same and different responses were approximately of the same speed. This finding might be explained by assuming that intervals longer than the standard were truncated and therefore less time was needed to make a different response than to make a same response. For the "short" condition,

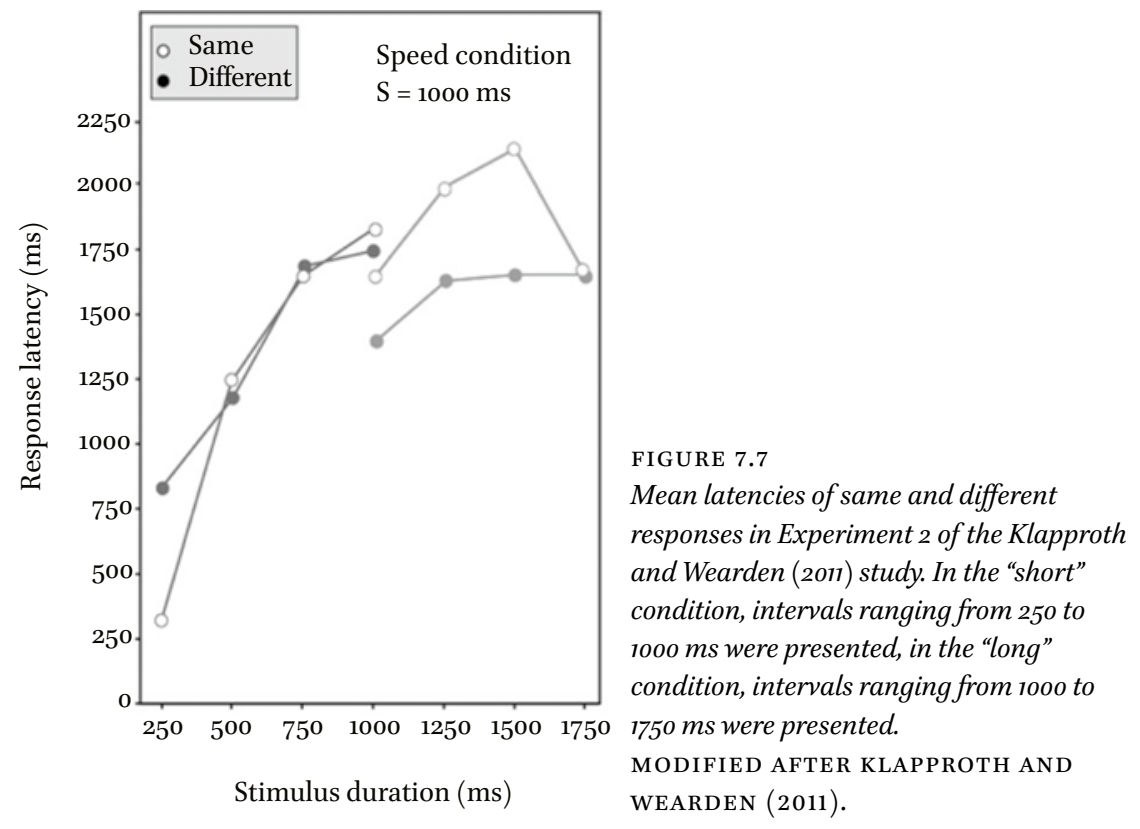


however, this explanation is not feasible since there was no interval longer than the standard. However, the probability model seems to be suitable for explaining the data from the "short" condition quite well. In this condition, $s^{*}$ should vary only between $s$ and zero, that is, it is not supposed to be larger than $s$. Consider now the case that a different response is made when the standard is presented. This would only be possible if $s^{*}$ is smaller than $s$. According to the model, fast-different responses are given when $t>s^{*}$, hence, different responses at $t=s$ should always be fast responses. The next case that is worth to be looked at is when a different response is made at an interval shorter than the standard. In that case, $s^{*}$ is either longer than the current interval or shorter. If it is longer, a slow-different response would occur, if it is shorter, a fastdifferent response would occur. Although the former case is more likely than the latter one, a mixture of fast and slow responses is expected which might contribute to a rather small discrepancy between the latencies of same and different responses.

According to the scalar timing theory, a same response will be given if $a b s(s-$ $t) / t<b$. The results of the speeded temporal-generalization experiments put forward the following question: How do the values of the representation of the standard duration, $s^{*}$, the representation of the presented duration, $t$, and the decision threshold, $b$, change when the participants make temporal judgments as quickly as possible?

\subsection{Changes of $s$}

From the experiments my colleagues and I conducted, it was inferred that the peak shift in temporal generalization under time pressure was presumably altered by the truncation of the longest stimuli of the series of stimulus durations. It was suggested that this truncation lowered the reference value of the standard duration. Thus, the $s$-value that was used for comparison with the current duration was not the same than the initial value of $s$. The experiments therefore suggest that $s$ cannot be simply regarded as the standard duration that is presented prior to the test trials or even within every test trial, but instead $s$ must be seen as a value that is strongly dependent on other durations that are experienced by the subject.

\subsection{Changes of $t$}

The $t$-value corresponds to the current duration, which is compared with the standard duration. However, it was shown that only durations smaller than or 
equal to the standard duration were fully considered for comparison. Comparison durations longer than the standard had presumably been truncated through premature responding. Therefore, the value of $t$ is not the same as the duration of the current interval, as long as the duration is larger than the standard. Instead, the maximum value of $t$ is supposed to be somewhat larger than the standard (according to the Klapproth and Müller, 2008, model: $t_{\max }=s / 1-b$ ). Since $t_{\max }$ is determined by the standard, and since intervals will be truncated after they had been presented for some time longer than the standard duration, the truncation in turn affects the value of $t_{\max }$, which is supposed to decrease when long stimuli are not experienced in their full length.

\subsection{Changes of $b$}

It has also been suggested that there is a special relationship between the $b$ value and response time. In one of their experiments, Klapproth and Wearden (2011) increased the difficulty of the generalization task by decreasing the stimulus spacing. As a result, the participants improved their discrimination performance and produced steeper gradients. According to the scalar timing theory, this should have been done through adapting a strict decision criterion, which corresponds to a rather low value of $b$. Moreover, in this experiment the participants not only made their decisions more precisely, but made them also more quickly. This seems counterintuitive at first glance, but is suitable to the model proposed for decision times in temporal generalization (Figure 7.1). In this model, the time to make a different response at long intervals is directly related to $b$ : the larger the value of $b$, the longer participants will need to make a different decision. This would also imply that large values of $b$ will allow larger intervals to be completely perceived than will small values of $b$. Therefore, the larger $b$ is, the smaller the truncation effect will be, and hence, the smaller will be the gradient's shift. Figure 7.8 illustrates the interdependency of premature responding and the alterations of the main parameters of the scalar timing theory.

\section{4}

\section{Conclusion}

What can we conclude from the findings reported in this chapter? First of all, the results imply that when intervals have to be judged within an experiment, the standard $s$ that is used for the comparative judgments seems to be not a fixed value, but rather dynamic. Its dynamic character results from all stimulus durations presented within the experiment, from which the mean of durations is supposed to form the reference duration. This "dynamic" representation of the reference duration has several implications for the judgment of subsequent 


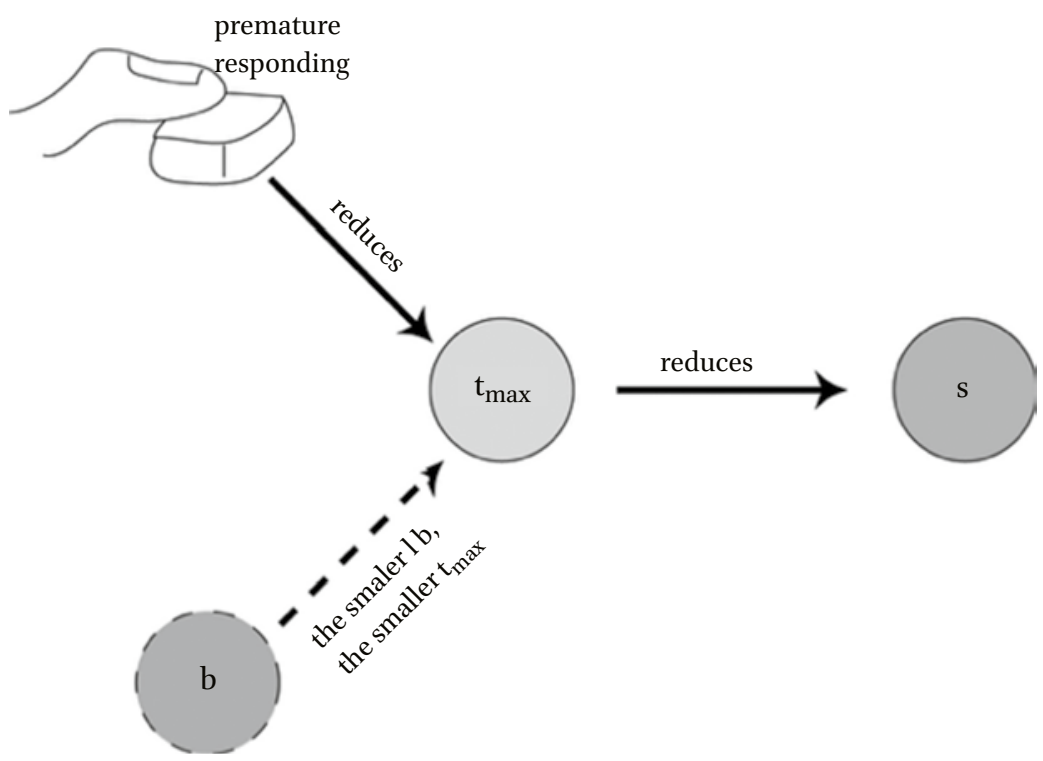

FIGURE 7.8 Synopsis of the effects of speeded duration judgment in temporal generalization on the timing parameters $\mathrm{t}, \mathrm{s}$, and $\mathrm{b}$.

(comparison) durations, and also for the scalar timing theory which often serves as a theoretical framework for comparative duration judgments. According to the scalar timing theory, duration judgments depend on at least three components of the judgment process, which are the reference (or standard) duration $s$, the to-be-judged (or comparison) duration $t$, and a decision threshold $b$. Since the value of $s$ depends on durations that were previously encountered within or across experimental trials, comparison durations would be judged as being larger, the shorter the previously encountered durations are. When individuals are encouraged to respond as quickly as possible, truncation of long comparison durations is likely to occur (resulting in a "shortening" of the encountered durations) so that subsequent comparison durations will be judged as being longer than they would be if the individuals take all time they need to make their judgments. As the standard $s$ depends on previous comparisons, and subsequent comparisons depend on the value of $s$, each single temporal comparison, where $s$ and $t$ were related to one another, depends on former comparisons and affects later comparisons. This exactly is the dynamic nature of the comparison process. The decision process and its outcomes are further affected by the decision threshold $b$, according to which a presented interval is categorized as either the standard duration or not the standard duration. If an individual applies a "strict" threshold, meaning that 
only small differences between the standard and comparison intervals are accepted for judging a comparison interval as equaling the standard, it is likely that long comparison durations are truncated. The truncation of long intervals, however, will result in lowering the value of the standard and hence in overestimation of subsequently presented comparison intervals. However, if the decision threshold is rather lax, truncation of comparison intervals is less likely, so that the respective standard is rather long, making overestimation of comparison intervals also less likely.

\section{References}

Balcı, F., \& P. Simen (2014). Decision processes in temporal discrimination. Acta Psychologica, 149, 157-168.

Desiderato, O. (1964). Effect of anxiety and stress on reaction time and temporal generalization. Psychological Reports, 14, 51-58.

Farell, B. (1985). Same-different judgments: A review of current controversies in perceptual comparisons. Psychological Bulletin, 98, 419-456.

Gallistel, C.R., \& J. Gibbon (2000). Time, rate, and conditioning. Psychological Review, 107, 289-344.

Gibbon, J. (1991). Origins of scalar timing. Learning and Motivation, 22, 3-38.

Gibbon, J., \& R.M. Church (1984). Sources of variance in information processing models of timing. In Roitblat, H.L., T.G. Bever, \& H.S. Terrace (Eds.), Animal cognition (pp. 465-488). Hillsdale, NJ: Lawrence Erlbaum Associates.

Gibbon, J., R.M. Church, \& W.H. Meck (1984). Scalar timing in memory. In Gibbon, J. \& L.G. Allan (Eds.), Timing and time perception (pp. $5^{2-77}$ ). New York, NY: New York Academy of Sciences.

Helson, H. (1948). Adaptation-level as a basis for a quantitative theory of frames of reference. Psychological Review, 55, 297-313.

Helson, H. (1964). Adaptation-level theory. New York, NY: Harper \& Row.

Klapproth, F., \& M. Müller (2008). Temporal generalization under time pressure in humans. Quarterly Journal of Experimental Psychology, 61, 588-60o.

Klapproth, F., \& J.H. Wearden (2011). Why do temporal generalization gradients change when people make decisions as quickly as possible? Quarterly Journal of Experimental Psychology, 64, 1646-1664.

Krueger, L.E. (1978). A theory of perceptual matching. Psychological Review, 85, 278-304.

Nickerson, R.S. (1975). Effects of correlated and uncorrelated noise on visual pattern matching. In Rabbitt, P.M.A. \& S. Domic (Eds.), Attention and performance (pp. 655668). London: Academic Press,. 
Pierrel, R., \& C.S. Murray (1963). Some relationships between comparative judgment, confidence, and decision-time in weight-lifting. American Journal of Psychology, 76 , $28-38$.

Posner, M.I., \& C.R.R. Snyder (1975). Facilitation and inhibition in the processing of signals. In Rabbitt, P.M.A. \& S. Domic (Eds.), Attention and performance (pp. 669-682). London: Academic Press.

Posner, M.I., R. Klein, J. Summers, \& S. Buggie (1973). On the selection of signals. Memory and Cognition, 1, 2-12.

Thomas, D.R. (1993). A model for adaptation-level effects on stimulus generalization. Psychological Review, 10o, 658-673.

Wearden, J.H. (1992). Temporal generalization in humans. Journal of Experimental Psychology: Animal Behavior Processes, 18, 134-144.

Wearden, J.H., L. Denovan, M. Fakhri, \& R. Haworth (1997). Scalar timing in temporal generalization in humans with longer stimulus durations. Journal of Experimental Psychology: Animal Behavior Processes, 23, 502-511.

Weisman, R., L. Brownlie, A. Olthof, M. Njegovan, C. Sturdy, \& D. Mewhort (1999). Timing and classifying brief acoustic stimuli by songbirds and humans. Journal of Experimental Psychology: Animal Behavior Processes, 25, 139-152. 